\title{
An Infant with Congenital Cytomegalovirus Infection Presenting with Hypomelanosis of Ito
}

\author{
Sinduja Selvam ${ }^{1}$, Amar Taksande², Amol Lohakare ${ }^{3}$, Rewat Meshram ${ }^{4}$ \\ 1, 2, 3,4 Department of Paediatrics, Jawaharlal Nehru Medical College, Sawangi, Maharashtra, India.
}

\section{INTRODUCTION}

Cytomegalovirus is the most common congenital viral infection in the world. Although the infection is largely asymptomatic, in the neonates the infection may result in significant neurodevelopment sequalae. It is the most common non-genetic cause of sensorineural hearing loss, which may be unilateral or bilateral and is usually progressive in nature. The infection is transmitted trans placentally and may occur in any trimester. The risk for transmission is maximum if the mother has a primary infection. These symptoms may range from IUGR (Intra-Uterine Growth Restriction), hydrops, vision loss, intellectual disability, cerebral palsy, epilepsy, autism, developmental delay, blueberry muffin baby, chorioretinitis, pneumonitis, microcephaly, thrombocytopenia, jaundice, petechiae, purpura. A majority of the babies are asymptomatic and may be one of the reasons for under recognition of the condition. The presence of hyperbilirubinemia in normal neonates and the lack of screening programs to identify the condition also contribute to under recognition. Early recognition and treatment with ganciclovir has been proven to reduce the developmental impacts and preserve hearing. ${ }^{1}$ Hypomelanosis of Ito is a rare disorder, characterized by non-progressive hypopigmentation, along the lines of Blaschko. The hypopigmentation may be unilateral or bilateral and is more common on the trunk and the extremities. The cause has been attributed to chromosomal anomalies in some and cutaneous mosaicism in others. This condition may be associated with abnormalities of the central nervous system or the musculoskeletal system. ${ }^{2-5}$ Here we report a case of a 5-month-old female child diagnosed with congenital cytomegalovirus infection on oral valganciclovir with unilateral hypomelanosis of Ito.

Congenital Cyto-Megalo-Virus infection (cCMV) is very common, yet the varied presentation makes the diagnosis challenging. However, early diagnosis for treatment with medication in symptomatic cases within the first month of life is critical. Hypomelanosis of Ito are less common manifestations at birth and may be overlooked. We present a case of an infant with congenital cytomegalovirus infection who presented with isolated hypopigmented patches, streaks and whorls, more over the left side of the body.

\section{PRESENTATION OF CASE}

A 5-month-old female child, a known case of CMV infection, presented with complaints of hypopigmented patches, streaks, and whorls, more prominent over the left side of the body, and failure to gain weight. The hypopigmentation was present since birth has not increased or changed in nature. The scalp, palms, and soles were relatively spared. The child was a product of non-consanguineous marriage, born at full term via normal delivery.
Corresponding Author: Dr. Sinduja Selvam, 1-D Jayapraksh Street, Kottur - 642114

Tamil Nadu, India.

E-mail: sinduja.th@gmail.com

DOI: $10.14260 / j e m d s / 2020 / 811$

How to Cite This Article:

Selvam S, Taksande A, Lohakare A, et al. An infant with congenital cytomegalovirus infection presenting with hypomelanosis of ito. J Evolution Med Dent Sci 2020;9(48): 3697-3699 DOI:

10.14260/jemds/2020/811

Submission 21-08-2020,

Peer Review 15-10-2020,

Acceptance 21-10-2020,

Published 30-11-2020.

Copyright (c) 2020 JEMDS. This is an open access article distributed under Creative Commons Attribution License [Attribution 4.0 International (CC BY 4.0)] 
There was no history of seizures or jaundice in the past or family history of skin disorders. The child had a history of blood transfusions, the first transfusion at about 12 days of life, and the rest at 2.5 months of age. The child was diagnosed to have CMV infection at 3 months of life and was started on oral valganciclovir. On examination, there was severe pallor, heart rate: 114 / $\mathrm{min}$, respiratory rate: 36 / $\mathrm{min}, \mathrm{Sp02} \mathrm{-} 98 \%$ on room air. She weighed $3500 \mathrm{~g}$, with a length of $57 \mathrm{~cm}$ and head circumference of $35 \mathrm{~cm}$, suggestive of acute on chronic malnutrition with no microcephaly. The anterior fontanelle was at level and pulsatile. The child had hypopigmented whorls and streaks which were more prominent over the extremities and hypopigmented patches over the trunk (Figure 1 \& Figure 2).
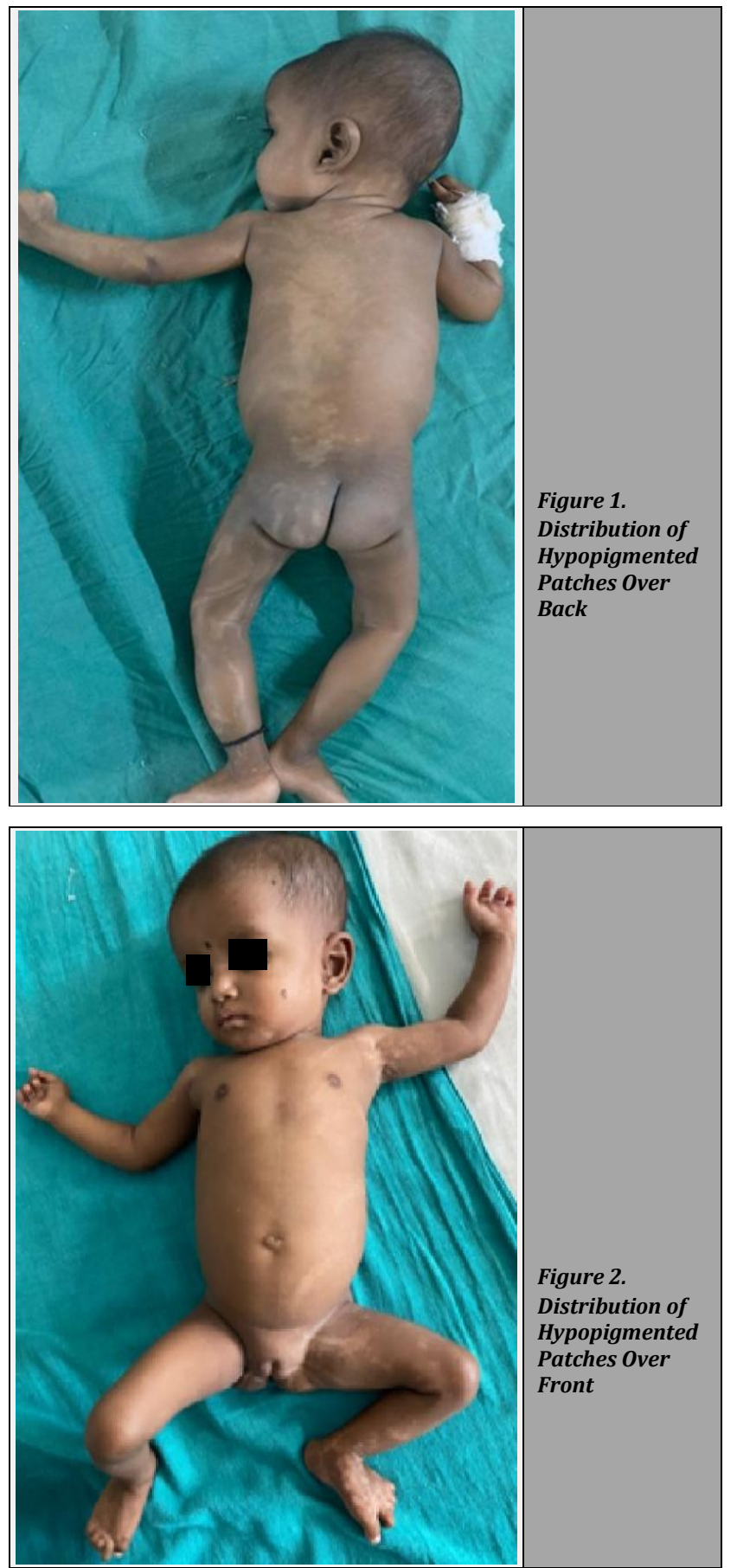

On systemic examination a systolic murmur was present over the parasternal area, air entry was equal and bilateral lungs were clear, liver was palpable $3 \mathrm{~cm}$ below the coastal margin, soft with regular borders and spleen was just palpable. Ultrasonography of abdomen and pelvis, 2 Dechocardiography as well as neurosonogram revealed no abnormality. Complete blood examination revealed severe microcytic hypochromic anaemia (Hb $2.5 \mathrm{gm} / \mathrm{dl}$ ). WBC 6500 per microliter and platelet count was 3.04 lakhs. HighPerformance Liquid Chromatography (HPLC) was normal. BERA (Brainstem Evoked Response Audiometry) done was of normal study. The child was given multiple aliquots of packed red blood cells. The child was discharged on oral valganciclovir, multivitamin supplementation, and advised to have a close follow-up.

\section{DISCUSSION}

Among congenital infection cytomegalovirus infection is the most common infection. The incidence is higher in the low socioeconomic groups and developing countries. Most of the neonates are asymptomatic at birth with about $10-15 \%$ presenting with multiple symptoms. ${ }^{1}$ Hypomelanosis is a rare condition with areas of non-progressive hypopigmentation, along the lines of Blaschko. It is also known as incontinentia pigmenti achromians or pigmentary mosaicism or dysplasia. The hypopigmentation presents as either unilateral or bilateral whorls, patches or lines, in early infancy and persists into childhood. The scalp, palms and soles, are usually not involved but it may be associated with refractory seizures, skeletal abnormalities and other abnormalities. The most common extra cutaneous anomalies involve the nervous system, like 'microcephaly or macrocephaly, cognitive and motor retardation, seizures, ataxia, hyperkinesia's and hypotonia'.6 Hence, it is also called neuro-cutaneous syndrome. Musculoskeletal disturbances manifest in more severe phenotypes with 'short stature, asymmetry like hemihypertrophy or clinodactyly, polydactyly, syndactyly, brachydactyly.7 Ocular involvement includes strabismus, nystagmus, hypertelorism, ptosis, myopia, amblyopia, cataracts, corneal opacity, microphthalmia, macrophthalmia, optic nerve hypoplasia and retinal degeneration. ${ }^{8}$ Among other systems, oral manifestation in the form of 'defective dental implantation, partial anodontia, dental hypoplasia or dysplasia, conical teeth and defective enamel' and kidney problems 'single kidney or ureteral duplication and genitourinary anomalies including cryptorchidism and micropenis' may also be associated with the condition. ${ }^{9}$ Neurocutaneous disorders like neurofibromatosis, tuberous sclerosis complex, Sturge-Weber syndrome, Von HippelLindau disease, incontinentia pigmenti and hypomelanosis of Ito primarily affect the skin and central nervous system but also affect several other organ systems and can be diagnosed bedside by an observant clinician. The child needs to be monitored for CNS manifestations during the follow-up period. The diagnosis is made based on clinical appearance and the history. The patient is managed with symptomatic and supportive treatment. Hypomelanosis of Ito is indistinguishable from systematized segmental nevus depigmentosus, a form of achromic nevus, in which the hypopigmentation patches, whorls and streaks are confined to one side of the body. ${ }^{7-10}$ The child should be followed up regularly for manifestations of the central nervous system. 
Financial or other competing interests: None.

Disclosure forms provided by the authors are available with the full text of this article at jemds.com.

\section{REFERENCES}

[1] Swanson EC, Schleiss MR. Congenital cytomegalovirus infection: new prospects for prevention and therapy. Pediatr Clin North Am 2013;60(2):335-49.

[2] Kumar HKY, Jayaprasad S. Hypomelanosis of ito: a rare cutaneous syndrome. Int J Health Allied Sci 2013;2(3):2035.

[3] Thapa R. Pigmentary mosaicism: an update. Indian J Dermatol 2008;53(2):96-7.

[4] Chethan CJ, Ashique KT, Sukumar D, et al. Hypomelanosis of ITO. Indian J Dermatol 2006;51(1):65-6.
[5] Ito M. A singular case of naevus depigmentosus systematicus bilateralis. Jpn J Dermatol 1951;61:31-2.

[6] Gomesz-Lado C, Eiris-Punal J, Barca-Blanco 0, et al. Hypomelanosis of Ito. A possibly under-diagnosed heterogeneous neurocutaneous syndrome. Rev Neurol 2004;38(3):223-8.

[7] Trägårdh $\mathrm{M}$, Thomsen $\mathrm{CR}$, Thorninger $\mathrm{R}$, et al. Hypomelanosis of Ito presenting with pediatric orthopedic issues: a case report. J Med Case Rep 2014;8:156.

[8] Weaver RG, Martin T, Zanolli MD. The ocular changes of incontinentia pigmenti achromians (hypomelanosis of Ito). J Pediatr Ophthalmol Strabismus 1991;28(3):160-3.

[9] Coward RJ, Risdon RA, Bingham C, et al. Kidney disease in hypomelanosis of Ito. Nephrol Dial Transplant 2001;16(6):1267-9.

[10] Di Lernia V. Segmental nevus depigmentosus: analysis of 20 patients. Pediatr Dermatol 1999;16(5):349-53. 